\title{
Tipología socio-ambiental de las ciudades medias de Brasil: aportes para un desarrollo urbano sostenible
}

\author{
Tipologia socioambiental de cidades médias no Brasil: \\ aportes para um desenvolvimento urbano sustentável
}
Socio-environmental typology of Brazilian medium size cities: contributions for a sustainable urban developtment

Fabio Angeoletto ${ }^{[a]}$, Jeater Waldemar Maciel Correa Santos ${ }^{[0]}$, Juan Pedro Ruiz Sanz ${ }^{[b]}$, Frederico Fonseca da Silva $a^{[c]}$, Ricardo Massulo Albertín ${ }^{[d]}$

\author{
[a] Universidade Federal de Mato Grosso (UFMT), Rondonópolis, MT, Brasil \\ [b] Universidad Autónoma de Madrid (UAM), Madrid, Espanha \\ [c] Instituto Federal do Paraná (IFPR), Curitiba, PR, Brasil \\ [d] Universidade Estadual de Maringá (UEM), Maringá, PR, Brasil
}

\section{Resumen}

La urbanización global de los territorios, que es un fenómeno ecológico humano importante en curso, y cuyos impactos ambientales poseen niveles local, regional y mundial, presenta la característica notable de un crecimiento pronunciado de las ciudades pequeñas e intermedias, en las cuales la capacidad de planificación y gestión es usualmente ínfima, en comparación a las ciudades grandes. Es lo que se verifica en Brasil, donde las ciudades de tamaño medio, es decir, las que tienen poblaciones entre 100.000 y 500.000 habitantes están creciendo más rápidamente que las grandes ciudades, desde mediados de la década de 1990. El artículo tiene como objetivo trazar una tipología de las ciudades medias brasileñas por medio de análisis estadísticos multivariados. La tipología resultante señala dos tipos de ciudades, con demandas sociales, ambientales y de gestión diferentes. Algunas de estas ciudades se encuentran en hotspots de biodiversidad. En paralelo, hemos recopilado estadísticas sobre la gestión ambiental en marcha en estas ciudades. Nuestros resultados demuestran una baja efectividad de las acciones de gestión ambiental en estos municipios.

Palabras clave: Ecología urbana. Gestión ambiental. Ciudades medias. Urbanización.

\section{Resumo}

A urbanização global dos territórios, que é um significativo fenômeno ecológico humano em curso e com impactos ambientais que possuem âmbito local, regional e global, apresenta como característica marcante um acentuado crescimento das cidades pequenas e médias, nas quais a capacidade de planejamento e de gestão é

FA: Profesor del Mestrado en Geografía de la Universidad Federal de Mato Grosso, campus de Rondonópolis, e-mail: fabio_angeoletto@yahoo.es

JWMCS: Profesor del Mestrado en Geografía de la Universidad Federal de Mato Grosso, campus de Rondonópolis, e-mail: jeater@ufmt.br

JPRS: Profesor del Doctorado en Ecología de la Universidad Autónoma de Madrid, e-mail: juan.ruiz@uam.es

FFS: Profesor del Instituto Federal de Paraná, e-mail: prof.frederico.silva@gmail.com

RMA: Doctorando em Geografia, Universidad Estadual de Maringá, e-mail: georrickk@yahoo.com.br 
usualmente ínfima em relação às grandes cidades. Essa tendência verifica-se no Brasil, onde as cidades médias, aquelas com populações entre 100 mil e 500 mil habitantes, têm crescido mais rapidamente do que as cidades grandes, desde meados dos anos 1990. O artigo teve como objetivo desenhar uma tipologia das cidades médias brasileiras por meio de análises estatísticas multivariadas. A tipologia resultante aponta dois tipos de cidades, com demandas socioambientais e de gestão diferentes. Parte dessas cidades está localizada em hotspots de diversidade biológica. Em paralelo, compilamos estatísticas sobre a gestão ambiental em curso nessas cidades. Nossos resultados apontam para uma baixa efetividade das ações de gestão ambiental nesses munícipios.

Palavras-chave: Ecologia urbana. Gestão ambiental. Cidades médias. Urbanização.

\section{Abstract}

The global urbanization of the territories, which is an important ongoing human ecological phenomenon, and whose environmental impacts have local, regional and global levels, has the remarkable property of a sharp growth of small and intermediate cities, in which capacity of planning and management is usually negligible, when they are compared to large cities. This is what takes place in Brazil, where medium-sized cities, those with populations between 100,000 and 500,000 residents are growing faster than large cities from mid-1990. The article aims to chart a typology of medium Brazilian cities, through multivariate statistical analysis. The resulting typology identifies two types of cities, with different social, environmental and management demands. Some of these cities are located in biodiversity hotspots. In parallel, we have compiled statistics on environmental management in place in these cities. Our results demonstrate low effectiveness of environmental management actions in these municipalities.

Keywords: Urban ecology. Environmental management. Medium cities. Urbanization.

Cities drive national economies by creating wealth, enhancing social development and providing employment, but they can also be the breeding grounds for poverty, exclusion and environmental degradation. (UN-HABITAT, 2008)

\section{Introducción}

La urbanización es uno de los más importantes procesos socio-ambientales de la actualidad. William Rees (1997) define la masiva migración de personas para las ciudades como el más significativo evento ecológico del siglo XX. También es consensual el enorme conjunto de impactos causados por ese proceso, a escala local, regional y global (Pickett et al., 1997; Terradas, 2001; Angeoletto, 2012). Según Vitousek (1994), la constante conversión de suelos en cultivos y ciudades es uno de los tres mayores impactos ambientales globales de origen humana, además de las crecientes concentraciones de $\mathrm{CO}^{2}$ en la atmosfera, y de otros cambios en los ciclos biogeoquímicos.
En las próximas décadas, la urbanización será el impacto humano globalmente más significativo, principalmente en los trópicos, si no se dan profundos cambios en políticas y planificación de los usos de suelo (Chapin III et al., 2010). La conversión de suelos relevantes, o bien desde el punto de vista social (suelos agrícolas) o bien desde el punto de vista ambiental (bosques, manglares y otros ecosistemas) es el impacto más negativo de la urbanización. De hecho, el espacio que ocupan los asentamientos urbanos está aumentando más rápidamente que la propia población urbana, un fenómeno común a las ciudades europeas, y que probablemente es asimismo global (Kasanko et al., 2006).

En 1900, solo un 10\% de la población global era urbana (Grimm et al., 2008). En 1950 la población 
urbana global subió a un 18\%, y en 2003 alcanzó el 42\% (WorldWatch Institute, 2006). En 2050 habrá entre ocho a diez mil millones de seres humanos (Cohen, 2003), 目 de los cuales habitarán en ciudades (Cohen, 2005). El 95\% del crecimiento de la población urbana ocurre en los países en desarrollo, cuyas ciudades reciben 5 millones de nuevos vecinos mensualmente (UN-HABITAT, 2008).

En Brasil, el proceso de urbanización resultó en un desplazamiento de millones de personas desde las zonas rurales hacia las ciudades. En 1890 la población urbana de Brasil era cerca de 10\%. En pocas décadas, ese cuadro se invierte: hoy, $83 \%$ de los brasileños viven en ciudades. En un primer ciclo, los emigrantes se han desplazado hacia las ciudades grandes.

Desde mediados de los años 90, se inicia un segundo ciclo de urbanización en Brasil, con un aumento considerable del número de ciudades medias (con poblaciones entre 100.000 y 500.000 habitantes), de personas viviendo en ellas y del área ocupada. Se puede observar nítidamente una disminución del área ocupada por las metrópolis, mientras que el total de las ciudades medias evoluciona, desde 1970 hasta 2000 , desde un $11,77 \%$ hasta un $27,23 \%$ del área total ocupada por las ciudades brasileñas. El número de ciudades medias también crece considerablemente en ese periodo, pasando de 40, en 1970, a 194, en 2000 (Carvalho, 2003).

Entre 2000 y 2010, esa expansión se mantuvo: mientras que las ciudades pequeñas y grandes crecieron a tasas anuales semejantes $(1,06 \%$ y $1,07 \%$, respectivamente), las ciudades medias crecieron 1,51\% al año (IPEA, 2011). Actualmente hay 283 ciudades medias en Brasil, que en conjunto concentran aproximadamente el $25 \%$ de la población brasileña (Angeoletto, 2012).

Las tendencias actuales de la urbanización brasileña reflejan un fenómeno global. Contrariamente a la creencia general, el grueso del aumento de la población urbana mundial ocurre en ciudades medianas y pequeñas, cuyas capacidades de planificación y gestión generalmente son débiles (UNFPA, 2007). En 2007, de los 3,3 mil millones de urbanitas, el 52\% vivían en ciudades con menos de 500 mil personas. Hasta 2025, esas ciudades van a absorber aproximadamente la mitad del crecimiento esperado de la población urbana global (United Nations, 2008). Además, los pobres constituirán una gran parte del futuro crecimiento poblacional urbano mundial (UNFPA, 2007).
Mientras las ciudades más pequeñas crecen, los núcleos familiares restan menores. Las familias han decrecido, entre 1970 y 2000 desde 5,1 a 4,4 personas/familia en los países en desarrollo, y desde 3,2 a 2,5 personas/familia en los países desarrollados.

Como la población mundial continua creciendo, familias más pequeñas significan más viviendas (Keilman, 2003). En Brasil, de 1991 a 2000, la media de familiares disminuyó de 4,95 personas/familia a 3,76 personas/familia, hecho que implicó la construcción de aproximadamente 4.630 .000 nuevas viviendas. En el ámbito mundial, se espera la construcción de 233 millones de viviendas, en hotspots de biodiversidad, para acomodar nuevos núcleos familiares (Liu et al., 2003).

Con relación al panorama de la urbanización brasileña, hemos trabajado para establecer una tipología de las tendencias socio-ambientales de las ciudades medias de Brasil y un cuadro de la efectividad de las acciones de gestión ambiental practicadas, con el objetivo de auxiliar al establecimiento de políticas ambientales urbanas más exitosas en la protección de la biodiversidad, y en el incremento de la calidad de vida de la gente.

\section{Algunas precisiones sobre fuentes y metodología de análisis}

Para explorar los estándares existentes en un conjunto de objetos o entidades de los cuales se hayan tomado mediciones de una manera analítica y cuantitativa, han sido creados diversos procedimientos estadísticos exploratorios, conocidos como "análisis multivariados" (Prado et al., 2001). Los análisis multivariados tienen como reto la reducción de un gran número de variables a pocas dimensiones, con un mínimo de pérdida de información, permitiendo la detección de los principales estándares de similitud, de asociación y de correlación entre dichas variables.

El método de la ordenación multivariada es cualquier proceso que proporcione un pequeño número de variables que describan las relaciones entre objetos a partir de mediciones tomadas de esos objetos (ciudades, por ejemplo) (Prado et al., 2001). El objetivo de la ordenación es reducir, sin pérdida de información, un gran número de mediciones a pocas variables sintéticas, facilitando la identificación de tendencias. En la mayoría de las técnicas de ordenación, 
las variables sintéticas, llamadas ejes o componentes, son combinaciones lineares de mediciones del tipo (Ecuación 1):

Ecuación 1:

$Y=a 1 x 1+a 2 x 2+a 3 x 3+\ldots a n x n$

Donde xi son las mediciones y ai sus coeficientes lineares que indican su importancia para el eje. La participación de cada medición en un eje puede también ser medida por su peso (loading), que indica su correlación con el eje. La coordenada de cada objeto en un eje es dado, como indica la fórmula, por Y, lo que permite usar los ejes en un plano cartesiano para representar relaciones multivariadas en pocas dimensiones (Prado et al., 2001).

La forma en que los ejes son calculados define cada técnica de ordenación, pero las más usadas en ciencias ambientales obedecen a dos restricciones importantes. La primera es que los ejes no estén correlacionados, de modo que cada eje capture tendencias independientes de los demás. Además de eso, los ejes poseen un orden de importancia en el que el primero es aquel que explica la mayor parte de la variación de los datos, y el segundo, aquel que explica la segunda mayor parte de la variación y así sucesivamente..

El análisis de componentes principales es, de los métodos de ordenación, el indicado para conjuntos de medidas correlacionados linealmente. Cuanto mayor es la correlación entre las mediciones originales, mayor será la proporción de variación explicada por los primeros ejes. Los coeficientes de las variables, o sus pesos, de mayor valor, ya sean positivos o negativos, son utilizados para interpretar los ejes (Barrasa, 2007).

También hemos procedido a un análisis estadístico univariado de los datos de gestión ambiental del universo de las ciudades investigadas, comparándolos a los datos de gestión de las ciudades seleccionadas a través del análisis de componentes principales. Las informaciones para los análisis estadísticos han sido obtenidas en dos bancos de datos: "Pesquisa de Informações Básicas Municipais - Perfil dos municipios brasileiros, meio ambiente 2002", disponibles en la página web del IBGE (2005b), (Instituto Brasileiro de Geografia e Estatistica), y a través de la página web IBGE Cidades (IBGE, 2014), ambas accedidas en 2014.
El banco de datos Perfil dos municipios brasileiros meio ambiente 2002 contiene informaciones sobre diferentes categorías de gestión ambiental (por ejemplo, gestión de recursos hídricos y gestión de recursos forestales), y su ocurrencia en las ciudades brasileñas. También están cuantificados datos institucionales como el número de funcionarios de las secretarias municipales de medio ambiente, y la prevalencia de impactos ambientales diversos, como episodios de contaminación atmosférica, deforestación y otros. El Perfil es el único documento producido por el IBGE con esta amplitud de datos relativos a cuestiones ambientales urbanas. En la página web IBGE Cidades hemos recolectado datos socioeconómicos de las ciudades medias investigadas.

Para el análisis de componentes principales, hemos compilado en una planilla del software Excel 127 variables, de las 258 ciudades medias de Brasil. Las variables han sido divididas del siguiente modo: variables ambientales (número de impactos ambientales, número de acciones de gestión ambiental), variables socioeconómicas (población, renta, escolaridad, coches privados, autobuses, motocicletas por grupos de 100.000 habitantes, entre otras).

La matriz de datos fue exportada al software estadístico SPSS donde se realizó un análisis de componentes principales (ACP). Con el ACP, hemos obtenido una tipología de las ciudades estudiadas, relacionándolas a sus sistemas de gestión ambiental y sus características socioeconómicas.

\section{Resultados y discusión}

El análisis de componentes principales revela dos grupos bien diferenciados, el de las ciudades pobres y ricas $^{1}$ (Cuadros 1 y 2; Figura 1). Esta información, recogida en los dos primeros ejes, explica el 77,95\% de varianza acumulada (un 57,19\% de varianza en el eje 1 ; y un $20,76 \%$ en el eje 2).

El primer grupo lo forman aquellas ciudades con valores negativos para el factor 1 y positivas para el factor 2, cuyas características más notables son un gran porcentaje de analfabetos, poblaciones jóvenes,

\footnotetext{
${ }^{1}$ Nos referimos a las ciudades seleccionadas a través del ACP como pobres y ricas, siempre señalándolas en cursiva, para resaltar las diferencias económicas entre ellas, aunque, evidentemente, existan distintas clases sociales en ambos grupos de ciudades.
} 
trabajadores con bajos sueldos y pocas acciones municipales de gestión ambiental. Este grupo de ciudades pobres está íntegramente concentrado en las regiones norte / noreste de Brasil, con la excepción de Águas Lindas de Goiás, localizada en la región Centro-Oeste, cerca de Brasilia, la capital federal brasileña.

Otra excepción notable es la presencia de la ciudad de Itapevi entre las más pobres, ya que ella está ubicada en el Estado de São Paulo, el más rico de Brasil, (las ciudades ricas están en las regiones sur y sureste). Se trata de ciudades que, a pesar de estar ubicadas cerca de regiones metropolitanas económicamente importantes, concentran pobreza e impactos ambientales correlacionados. Esas ciudades reproducen, así, el fenómeno común en regiones metropolitanas brasileñas: una relativa prosperidad en las ciudades-polo de las regiones, faz la escasez de recursos y varias otras inequidades (incluso de matiz ambiental) en las ciudades del entorno.

Por otro lado, las ciudades con valores positivos para factor 1 son las ciudades ricas, con poblaciones adultas y escolarizadas, con gran proporción de vehículos por habitante, y con más acciones de gestión ambiental (Cuadro 2).

En oposición a las ciudades pobres del norte y noreste de Brasil, en las ciudades ricas predominan poblaciones adultas, con bajas tasas de natalidad, $\mathrm{y}$ además, más escolarizadas y con sueldos mayores. Por lo tanto, con estándares de consumo más sofisticados que aquellos presentes en las ciudades pobres.

Los coches y otros vehículos ilustran bien las diferencias de consumo entre las ciudades medias pobres y ricas de Brasil. Las ciudades del sur y sudeste de Brasil tienen una relación vehículos por habitante muy similar a las de las ciudades de los

Cuadro 1 - Ciudades pobres del ACP

Cuadro 2 - Ciudades ricas del ACP

\begin{tabular}{|c|c|c|c|c|c|}
\hline Ciudades pobres & Bioma & Región & Ciudades ricas & Bioma & Región \\
\hline Abretetuba & Amazônia & Norte & Araçatuba & Mata Atlântica & Sureste \\
\hline Açailândia & Amazônia & Noreste & Bento Gonçalves & Mata Atlântica & Sur \\
\hline Cametá & Amazônia & Norte & Blumenau & Mata Atlântica & Sur \\
\hline Castanhal & Amazônia & Norte & Catanduva & Mata Atlântica & Sureste \\
\hline Macapá & Amazônia & Norte & Caxias do Sul & Mata Atlântica & Sur \\
\hline Marabá & Amazônia & Norte & Florianópolis & Mata Atlântica & Sur \\
\hline Parintins & Amazônia & Norte & Jundiaí & Mata Atlântica & Sureste \\
\hline Santarém & Amazônia & Norte & Maringá & Mata Atlântica & Sur \\
\hline São José de Ribamar & Amazônia & Noreste & Niterói & Mata Atlântica & Sureste \\
\hline Águas Lindas de Goiás & Cerrado & Centro-Oeste & Poços de Caldas & Mata Atlântica & Sureste \\
\hline Caxias & Cerrado & Noreste & Presidente Prudente & Mata Atlântica & Sureste \\
\hline Codó & Cerrado & Noreste & Santos & Mata Atlântica & Sureste \\
\hline Timon & Cerrado/Caatinga & Noreste & São Vicente & Mata Atlântica & Sureste \\
\hline Caucaia & Caatinga & Noreste & Vitória & Mata Atlântica & Sureste \\
\hline Itapipoca & Caatinga & Noreste & Santa Cruz do Sul & Mata Atlântica/Pampa & Sur \\
\hline Arapiraca & Caatinga/ Mata Atlântica & Noreste & Americana & Mata Atlântica/Cerrado & Sureste \\
\hline Itapevi & Mata Atlântica & Sureste & Bauru & Mata Atlântica/Cerrado & Sureste \\
\hline Nossa Senhora do Socorro & Mata Atlântica & Noreste & Botucatu & Mata Atlântica/Cerrado & Sureste \\
\hline Santa Rita & Mata Atlântica & Noreste & Rio Claro & Mata Atlântica/Cerrado & Sureste \\
\hline Simões Filho & Mata Atlântica & Noreste & São José do Rio Preto & Mata Atlântica/Cerrado & Sureste \\
\hline Fuente: elaboración propia. & & & Fuente: elaboración propia. & & \\
\hline
\end{tabular}

urbe. Revista Brasileira de Gestão Urbana (Brazilian Journal of Urban Management), 2016 maio/ago., 8(2), $272-287$ 


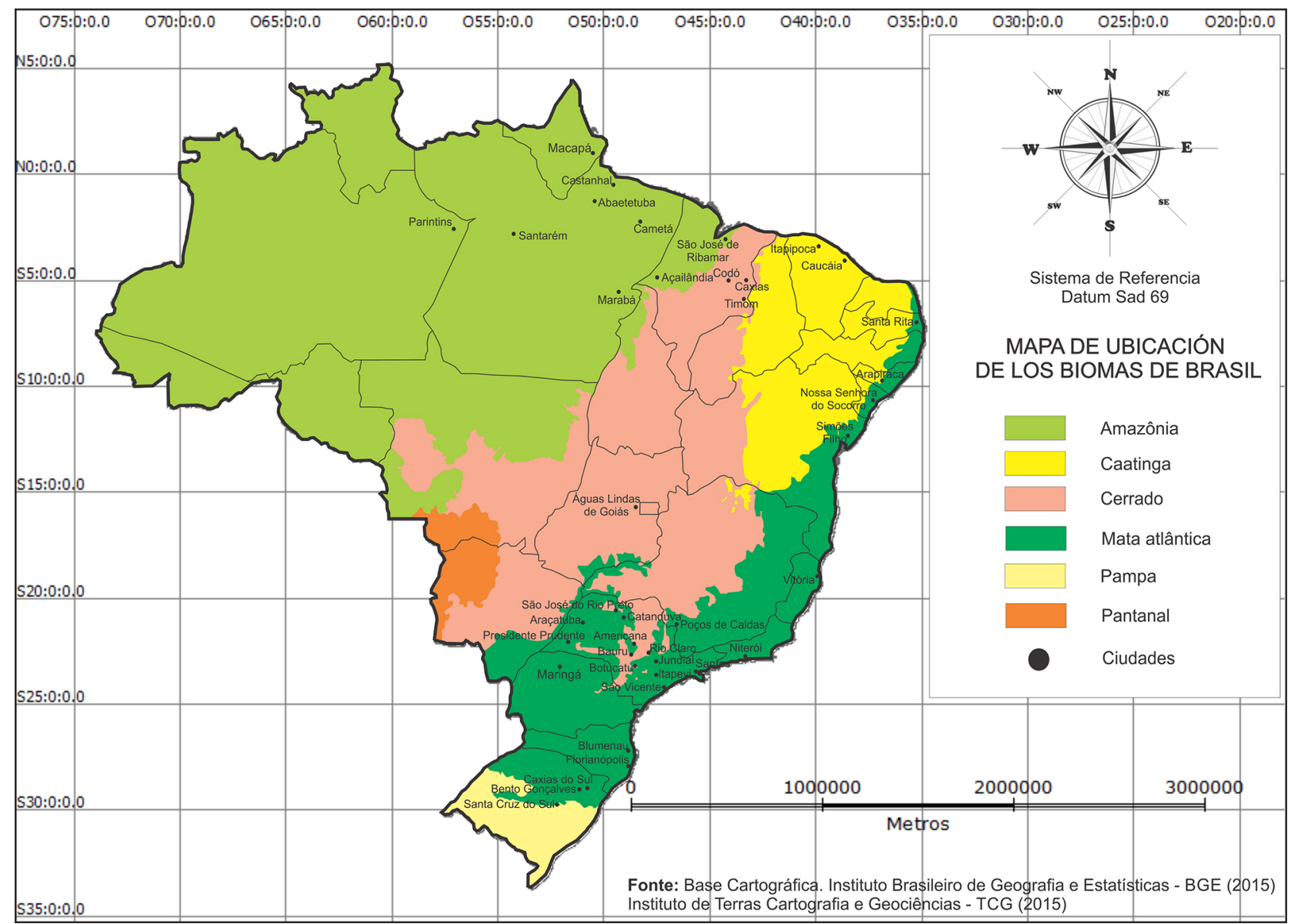

Figura 1 - Ubicación biogeográfica de las ciudades seleccionadas en el ACP Fuente: Base Cartográfica. Instituto Brasileiro de Geografia e Estatísticas - IBGE (2015); Instituto de Terras, Cartografia e Geociências - ITCG (2015).

países del primer mundo. En la ciudad de Maringá, por ejemplo, esa relación es de un vehículo privado para cada 2,08 habitantes. Codó, una de las ciudades pobres de nuestro análisis, tiene una relación de un vehículo para cada 17,71 habitantes y Cametá tiene un vehículo para cada 55,1 habitantes (Cuadros 3 y 4).

La motorización y sus impactos en las ciudades medias brasileñas

Aproximadamente dos tercios de la flota mundial de vehículos están compuestos por coches privados, de los que actualmente se fabrican aproximadamente 32 millones al año (lo que significa uno por segundo). La gran capacidad del coche privado de contaminar llevó a la Unión Europea a considerar el transporte como la causa individual más importante del daño medioambiental (Bettini, 1998).
Atascos, atropellos, mutilaciones y muertes; contaminación generalizada, fragmentación de ecosistemas, daños a la salud pública, muertes de animales en las carreteras. La relación de impactos socio-ambientales provocados por un modelo de transporte excesivamente basado en vehículos privados es extensa. En Brasil, los vehículos de las ciudades con más de 60.000 habitantes consumen aproximadamente 10 millones de toneladas equivalentes de petróleo. Además, en esas ciudades, el transporte individual es responsable por $74 \%$ del consumo de energía y del $80 \%$ de las emisiones de contaminantes nocivos para la salud humana. Los vehículos motorizados emiten 24 millones de toneladas de contaminantes anualmente, siendo el coste estimado de la contaminación de cerca de 1.600 millones de euros, y el transporte privado responsable del 86\% de este valor (Brasil, 2004).

La baja relación ciudadanos/vehículo que hemos encontrado en las ciudades medias más prósperas apuntadas por nuestro análisis de componentes 
Cuadro 3 - Relación vecinos/vehículos privados en las ciudades ricas

\begin{tabular}{lc}
\hline \multicolumn{1}{c}{ Ciudad } & Relación Vecinos/Vehículos \\
\hline Catanduva & 1,98 \\
Jundiaí & 2,07 \\
Maringá & 2,08 \\
Blumenau & 2,15 \\
Bento Goncálves & 2,16 \\
São José do Rio Preto & 2,18 \\
Americana & 2,19 \\
Aracatuba & 2,21 \\
Rio Claro & 2,23 \\
Santa Cruz do Sul & 2,27 \\
Florianópolis & 2,33 \\
Santos & 2,37 \\
Botucatu & 2,47 \\
Pocos de Caldas & 2,52 \\
Presidente Prudente & 2,59 \\
Caxias do Sul & 2,68 \\
Bauru & 2,74 \\
Vitórí & 2,86 \\
Niterói & 3,02 \\
São Vicente & 6,66 \\
\hline
\end{tabular}

Fuente: elaboración propia, a partii de datos del IBGE Cidades (BBGE, 2014).

principales supone un importante problema de salud pública, por la contaminación emitida por vehículos (y la plétora de otros impactos asociados a su uso). Además, esos datos sugieren claramente que son necesarias medidas para la reducción del papel del coche, preponderante no sólo en el transporte sino también en la conformación de la morfología de las ciudades de Brasil.

De acuerdo con el arquitecto Virginio Bettini (1998), el sistema de transporte público es la piedra fundamental de cualquier ciudad que desea ser "ecológicamente correcta", aunque no sea simple afrontar el papel prevaleciente del automóvil en las ciudades. Pocas son las inversiones en transporte público en comparación con las destinadas al transporte privado: los impuestos que todos pagan son invertidos en infraestructura para una minoría de propietarios de coches en las ciudades brasileñas. El sesgo hacia los propietarios de vehículos, en una planificación enfocada solamente en el aumento del flujo de
Cuadro 4 - Relación vecinos/vehículos privados en las ciudades pobres

\begin{tabular}{lc}
\hline \multicolumn{1}{c}{ Ciudades } & Relación Vecinos/Vehículos \\
\hline Arapiraca & 5,86 \\
Itapevi & 6,26 \\
Macapó & 6,43 \\
Marabá & 6,91 \\
Castanhal & 7,63 \\
Santarém & 9,85 \\
Simões Filho & 9,94 \\
Itapipoca & 10,03 \\
Acailândia & 10,15 \\
Caxios & 11,03 \\
Santa Rita & 13,21 \\
Nossa Senhora do Socorro & 13,88 \\
Caucaia & 15,25 \\
Parintins & 15,38 \\
Codó & 17,71 \\
Timon & 19,28 \\
Abaetetubba & 24 \\
Águas Lindas de Goiás & 25,27 \\
São José de Ribamar & 29,74 \\
Cametó & 55,11 \\
\hline
\end{tabular}

Fuente: elaboración propia, a partir de datos del IBGE Cidades (IBGE, 2014).

vehículos, y que ignora impactos socio-ambientales, es usual en Brasil.

A diferencia de las décadas anteriores, hoy en las ciudades brasileñas, las fuentes móviles (es decir, vehículos con motores de combustión interna) son las principales emisoras de contaminación atmosférica. Incluso en Sao Paulo, la megalópolis más industrializada de Brasil (y una de las más industrializadas del planeta) son los coches y otros vehículos las más importantes fuentes emisoras de contaminantes atmosféricos (Saldiva et al., 1994; Martins et al., 2001). Oliveira \& Leitmann (1994) y Leitmann (1995) estimaron que los vehículos contribuyen, para la ciudad de São Paulo, con un porcentaje entre $73 \%$ y $94 \%$ de los principales contaminantes atmosféricos normalmente encontrados en áreas urbanas.

En las ciudades latinoamericanas, son los vehículos los mayores contaminadores del aire (CEPAL, 2000; ECLAC, 2001). El mismo fenómeno se verifica en otras partes. En Atenas, más de $80 \%$ de la contaminación 
atmosférica viene de los coches (Mega, 2000), y en la Ciudad de México ese porcentaje alcanza un 76,7\% (Garza, 1996). Mundialmente el $42 \%$ del gas invernadero $\mathrm{CO}^{2}$ es emitido por vehículos (Bettini, 1998).

Se pueden resumir las consecuencias de la contaminación producida por vehículos para los ecosistemas urbanos (la motorización impone también impactos regionales y globales, como señalan, por ejemplo, Silva et al., 2003) en dos campos: enfermedades diversas y daños a la biodiversidad urbana. Por ejemplo, el contaminante NOx reacciona con compuestos orgánicos volátiles, resultando en la formación de ozono, que es tóxico para las plantas, animales y seres humanos (Botkin \& Beveridge, 1997; Martins et al., 2002).

Factores como estos indican la complejidad de planificar los ecosistemas urbanos. Alcanzar ciertos estándares de negentropía dentro de los límites urbanos no solamente es una cuestión tecnológica, como nos quieren hacer creer algunos ingenieros e intelectuales optimistas en la capacidad humana para el progreso tecnológico. Las ciudades medias más motorizadas apuntadas por el ACP tienen, efectivamente, un desafío de una gran magnitud, que comprende intersecciones entre salud pública, protección medioambiental, planificación y gestión ambiental urbana.

En realidad, en mayor o menor grado, es este un dilema de todas las ciudades medias de Brasil, dado que hay una rápida y creciente expansión de la flota nacional, que se puede explicar por diversos factores, entre ellos, estabilización de la economía, carencia de planeamiento del uso del suelo urbano, adopción de un modelo de transporte que prioriza los desplazamientos en vehículos privados de combustión interna y una preocupante carencia de inversión en el transporte público de masas (Vianna, et al., 2004) además de incentivos gubernamentales a la compra de vehículos a través de la reducción del IPI (imposto sobre productos industrializados) sobre la venta de coches y motocicletas, como una respuesta a la crisis económica de 2008.

La gestión ambiental en ciudades medias de Brasil - cuadro institucional

De las ciudades ricas apuntadas en el análisis de componentes principales, el 50\% poseían secretaría de medio ambiente exclusiva, o sea, no vinculada a otras secretarías, y por tanto dedicadas exclusivamente a las cuestiones medioambientales. En el grupo de las ciudades pobres del ACP, ese porcentaje decrece a un 15\%. Para el universo de las 283 ciudades analizadas, sorprendentemente, solo un $25 \%$ de los municipios poseían secretarias de medio ambiente exclusivas.

Las ciudades ricas poseen, proporcionalmente, más funcionarios actuando en cuestiones ambientales. Cuatro de las 20 ciudades pobres seleccionadas en nuestro estudio no tienen siquiera un único funcionario destinado al medio ambiente. No obstante, los funcionarios disponibles son pocos para ambos grupos de ciudades investigadas (Cuadros 5 y 6). Para la muestra de las ciudades investigadas, en número medio de funcionarios era de 13 por grupo de 100.000 habitantes, siendo de esos, dos con carrera universitaria.

Un 60\% de los municipios pobres tenía legislación ambiental específica para cuestiones ambientales, porcentaje que alcanzaba un $80 \%$ en las ciudades ricas. En el conjunto de ciudades analizadas, un 78\% de ellas poseían legislación ambiental específica para cuestiones ambientales.

\section{Cuadro 5 - Número de funcionarios municipales destinados a medio ambiente (las 10 más)}

\begin{tabular}{lc}
\hline $\begin{array}{c}\text { Número total de } \\
\text { funcionarios/100.000 } \\
\text { habitantes }\end{array}$ & $\begin{array}{c}\text { Funcionarios con carrera } \\
\text { universitaria/100.000 } \\
\text { habitantes }\end{array}$ \\
\hline Baurv: 58,21 & 1,58 \\
Vitória: 51 & 8,89 \\
Catanduva: 43,52 & Cero \\
Presidente Prudente: 41,75 & 0,53 \\
Florianópolis: 30,11 & 1,75 \\
Araçatutuba: 20,11 & 1,77 \\
Santos: 22,51 & 6,22 \\
Caxias do Sul: 19,7 & 1,94 \\
Jundiaí: 10,5 & 6,21 \\
São José do Rio Preto & 4,22 \\
\hline
\end{tabular}

Fuente: IBGE: Perfil dos Municípios Brasileiros - Meio Ambiente (IBGE, 2005a) y elaboración propia. 
Acciones de gestión ambiental (fiscalización)

Las acciones de fiscalización (resumidas en el Cuadro 7), en general, ocurren en un porcentaje reducido de las ciudades pobres seleccionadas por el

Cuadro 6 - Número de funcionarios municipales destinados a medio ambiente (las 10 menos)

\begin{tabular}{lc}
\hline $\begin{array}{c}\text { Número total de fun- } \\
\text { cionarios/100.000 } \\
\text { habitantes }\end{array}$ & $\begin{array}{c}\text { Funcionarios con carrera } \\
\text { universitaria/100.000 } \\
\text { habitantes }\end{array}$ \\
\hline Macapá: 25,77 & 1,4 \\
Arapiraca; 18,77 & 0,54 \\
Cametá: 18,07 & Cero \\
Santarém: 10,28 & 1,9 \\
Timon 6,73 & Cero \\
São José de Ribamar: 2,37 & Cero \\
Parintins; cero & Cero \\
Castanhal: cero & Cero \\
Codó: cero & Cero \\
Itapipoca: cero & Cero \\
\hline Fuente: IBGE: Perfil dos Municípios Brasileiros - Meio Ambiente (IBGE, 2005a) y \\
elaboración propia.
\end{tabular}

análisis de componentes principales, en comparación con las ciudades ricas y el universo de ciudades estudiadas. No obstante, tres categorías de fiscalización se destacan por su baja ocurrencia, cualquiera que sea el grupo de ciudades: la fiscalización sobre la contaminación atmosférica producida por vehículos, la fiscalización de gasolineras, y lafiscalización sobre el uso de fertilizantes y pesticidas en cultivos agrícolas.

Esas acciones resultaron, en el año que los datos fueron recogidos por el IBGE, en medidas judiciales o administrativas contra ciudadanos, empresas o instituciones que provocaron impactos ambientales en un $20 \%$ de las ciudades pobres y $75 \%$ de las ciudades ricas del ACP; y en $48 \%$ del total de las ciudades medias investigadas. A su vez las multas aplicadas por impactos ambientales provocados por ciudadanos, empresas o instituciones oscilaron entre el $15 \%$ y $70 \%$ en las ciudades pobres y ricas del ACP y fueron aplicadas en $63 \%$ del conjunto de las 283 ciudades estudiadas.

Cuadro 7 - Acciones de gestión ambiental (fiscalización)

\begin{tabular}{|c|c|c|c|c|}
\hline $\begin{array}{l}\text { Acciones } \\
\text { de gestión: } \\
\text { fiscalización }\end{array}$ & $\begin{array}{c}\text { Tipo de } \\
\text { fiscalización }\end{array}$ & $\begin{array}{c}\text { Porcentaje } \\
\text { de ocurrencia } \\
\text { (ciudades pobres } \\
\text { del } \mathrm{ACP}, \mathrm{N}=20 \text { ) }\end{array}$ & $\begin{array}{c}\text { Porcentaje } \\
\text { de ocurrencia } \\
\text { (ciudades pobres } \\
\text { del } \mathbf{A C P}, \mathbf{N}=20 \text { ) }\end{array}$ & $\begin{array}{c}\text { Porcentaje } \\
\text { de ocurrencia } \\
\text { (universo de } \\
\text { las ciudades } \\
\text { investigadas, } \\
\mathbf{N}=\mathbf{2 8 3} \text { ) }\end{array}$ \\
\hline & $\begin{array}{l}\text { Fiscalización sobre la disposición } \\
\text { irregular de residuos domésticos }\end{array}$ & $50 \%$ & $80 \%$ & $72,1 \%$ \\
\hline & $\begin{array}{l}\text { Fiscalización sobre la disposición } \\
\text { irregular de residuos industriales }\end{array}$ & $45 \%$ & $75 \%$ & $62,2 \%$ \\
\hline & $\begin{array}{l}\text { Fiscalización sobre actividades } \\
\text { industriales contaminantes }\end{array}$ & $25 \%$ & $60 \%$ & $55,12 \%$ \\
\hline & $\begin{array}{l}\text { Fiscalización sobre actividades } \\
\text { extractivas }\end{array}$ & $30 \%$ & $55 \%$ & $53 \%$ \\
\hline & $\begin{array}{l}\text { Fiscalización sobre contaminación } \\
\text { producida por vehículos }\end{array}$ & $10 \%$ & $20 \%$ & $9,89 \%$ \\
\hline & Fiscalización de gasolineras & $30 \%$ & $45 \%$ & $53 \%$ \\
\hline & $\begin{array}{l}\text { Fiscalización de áreas naturales } \\
\text { legalmente protegidas }\end{array}$ & $40 \%$ & $85 \%$ & $62,9 \%$ \\
\hline & $\begin{array}{l}\text { Fiscalización sobre el uso de } \\
\text { fertilizantes y pesticidas }\end{array}$ & $15 \%$ & $30 \%$ & $26,15 \%$ \\
\hline
\end{tabular}

Fuente: IBGE: Perfil dos Municípios Brasileiros - Meio Ambiente (IBGE, 2005a) y elaboración propia. 
Acciones de gestión ambiental

(protección de recursos naturales)

Respecto a las acciones municipales de protección de recursos naturales (Cuadro 8), son especialmente preocupantes los bajos porcentuales de ciudades que practicaron la monitorización de la calidad y descontaminación del agua de cuerpos hídricos. Acciones de protección a los suelos agrícolas virtualmente no existieron. Las acciones de control de la deforestación fueron frecuentes solamente en las ciudades ricas del ACP. Pocas fueron las ciudades que habían elaborado sus planes de zonificación ecológico-económico, hecho que indica un bajo esfuerzo de planificación.
Programas de medio ambiente municipales

La gran mayoría de las ciudades ricas del ACP (el 85\%) habían desarrollado programas de recogida selectiva de basura reciclable, aunque el IBGE no informe el porcentaje de basura efectivamente captada por esos programas. Asimismo ha sido elevado el porcentaje de ciudades (el 68\%) donde se han desarrollado programas de educación ambiental, en el universo investigado. El desarrollo de programas agrícolas de control biológico de plagas y de producción de alimentos sin el uso de pesticidas y fertilizantes industriales (dos grandes fuentes de impactos ambientales) ha sido bajo, incluso en las ciudades ricas apuntadas a través del análisis de componentes principales (Cuadro 9).

Cuadro 8 - Acciones de gestión ambiental (protección de recursos naturales)

\begin{tabular}{|c|c|c|c|c|}
\hline $\begin{array}{l}\text { Acciones } \\
\text { de gestión: } \\
\text { protección } \\
\text { de recursos } \\
\text { naturales }\end{array}$ & Tipo de acción & $\begin{array}{c}\text { Porcentaje } \\
\text { de ocurrencia } \\
\text { (ciudades pobres } \\
\text { del } \mathbf{A C P}, \mathbf{N}=20 \text { ) }\end{array}$ & $\begin{array}{c}\text { Porcentaje } \\
\text { de ocurrencia } \\
\text { (ciudades pobres } \\
\text { del } \mathrm{ACP}, \mathrm{N}=20 \text { ) }\end{array}$ & $\begin{array}{c}\text { Porcentaje } \\
\text { de ocurrencia } \\
\text { (universo de } \\
\text { las ciudades } \\
\text { investigadas, } \\
\mathbf{N}=\mathbf{2 8 3} \text { ) }\end{array}$ \\
\hline \multirow[t]{3}{*}{$\begin{array}{l}\text { Protección de los recursos } \\
\text { hídricos }\end{array}$} & $\begin{array}{l}\text { Mejorías en el sistema de colecta de } \\
\text { desechos }\end{array}$ & $45 \%$ & $75 \%$ & $64 \%$ \\
\hline & $\begin{array}{l}\text { Acciones de descontaminación de cuerpos } \\
\text { hídricos }\end{array}$ & Cero & $50 \%$ & $27,92 \%$ \\
\hline & $\begin{array}{l}\text { Monitorización de la calidad del agua de } \\
\text { cuerpos hídricos }\end{array}$ & Cero & $50 \%$ & $17 \%$ \\
\hline \multirow{3}{*}{$\begin{array}{l}\text { Protección de recursos } \\
\text { forestales }\end{array}$} & Control de la deforestación & $20 \%$ & $75 \%$ & $43,11 \%$ \\
\hline & Control de quemadas & $30 \%$ & $45 \%$ & $30,04 \%$ \\
\hline & $\begin{array}{l}\text { Acciones de recomposición de áreas } \\
\text { deforestadas }\end{array}$ & $25 \%$ & $90 \%$ & $56,2 \%$ \\
\hline \multirow{3}{*}{$\begin{array}{l}\text { Acciones de protección a suelos } \\
\text { agrícolas }\end{array}$} & Control de la salinidad del suelo & Cero & $5 \%$ & $1,1 \%$ \\
\hline & Combate a la erosión & $10 \%$ & $60 \%$ & $33,92 \%$ \\
\hline & $\begin{array}{l}\text { Acciones de recuperación de áreas degra- } \\
\text { dadas por actividades agropecuarias }\end{array}$ & $15 \%$ & $20 \%$ & $20,85 \%$ \\
\hline \multirow[t]{2}{*}{$\begin{array}{l}\text { Acciones de ordenamiento } \\
\text { territorial }\end{array}$} & $\begin{array}{l}\text { Monitorización de la ocupación de suelo } \\
\text { urbano }\end{array}$ & $45 \%$ & $90 \%$ & $71,02 \%$ \\
\hline & Plan de zonificación ecológico-económico & $10 \%$ & $30 \%$ & $18,02 \%$ \\
\hline
\end{tabular}

Fuente: IBGE: Perfil dos Municípios Brasileiros - Meio Ambiente (IBGE, 2005a) y elaboración propia. 
Presencia de equipos para la gestión ambiental urbana

En cuanto a los equipos para la gestión ambiental, la situación retratada por IBGE, era, en el año de la recolección de datos, absolutamente preocupante. Con la excepción de la existencia de vertederos sanitarios (que faltaban en nada menos que el $42 \%$ del conjunto de las 283 ciudades investigadas), los demás equipos han sido detectados en porcentuales aún más modestos. Lo mismo se verificó, también, respecto a la presencia de sitios para recogida de envases de pesticidas, aunque el consumo de este insumo agrícola fuera significativo en respectivamente $65 \%$ y $75 \%$ de las ciudades pobres y ricas seleccionadas por el análisis de componentes principales.

En relación al conjunto de ciudades investigadas, el consumo de pesticidas es significativo en $70 \%$ de los municipios. De modo semejante, pocas ciudades poseían vertederos para el recibimiento adecuado de residuos industriales, aunque la producción de residuos fuera significativa en $65 \%$ de las ciudades pobres del ACP; en $90 \%$ de las ciudades ricas; y en $69 \%$ del total de ciudades analizadas. Solamente un 15\% de los municipios pobres poseían unidades de conservación municipales (como parques y bosques), contra un $95 \%$ entre las ciudades ricas señaladas por el ACP. Por otro lado, la presencia de estaciones de medición de la calidad del aire no era común en los tres grupos de ciudades medias analizadas (Cuadro 10).

La escasez de funcionarios dedicados al medio ambiente, así como la relativa escasez de equipos y acciones de gestión ambiental en las ciudades medias de Brasil, sugieren un cuadro de poca efectividad ante los problemas e impactos ambientales que necesitan ser corregidos o evitados en esas ciudades. El cuadro

Cuadro 9 - Acciones de gestión ambiental (programas ambientales)

\begin{tabular}{|c|c|c|c|c|}
\hline $\begin{array}{l}\text { Acciones } \\
\text { de gestión: } \\
\text { programas } \\
\text { ambientales }\end{array}$ & $\begin{array}{l}\text { Tipo de } \\
\text { programa }\end{array}$ & $\begin{array}{c}\text { Porcentaje } \\
\text { de ocurrencia } \\
\text { (ciudades pobres } \\
\text { del } \mathbf{A C P}, \mathbf{N}=\mathbf{2 0} \text { ) }\end{array}$ & $\begin{array}{c}\text { Porcentaje } \\
\text { de ocurrencia } \\
\text { (ciudades pobres } \\
\text { del } \mathbf{A C P}, \mathbf{N}=\mathbf{2 0} \text { ) }\end{array}$ & $\begin{array}{c}\text { Porcentaje de } \\
\text { ocurrencia (universo } \\
\text { de las ciudades } \\
\text { investigadas, } N=283 \text { ) }\end{array}$ \\
\hline & $\begin{array}{l}\text { Recogida selectiva de } \\
\text { basura reciclable }\end{array}$ & $10 \%$ & $85 \%$ & $54,1 \%$ \\
\hline & Educación ambiental & $40 \%$ & $75 \%$ & $67,84 \%$ \\
\hline & $\begin{array}{l}\text { Control biológico de plagas } \\
\text { agrícolas }\end{array}$ & $10 \%$ & $25 \%$ & $21,91 \%$ \\
\hline & $\begin{array}{l}\text { Incentivo a prácticas de } \\
\text { agricultura orgánica }\end{array}$ & $25 \%$ & $40 \%$ & $35 \%$ \\
\hline
\end{tabular}

Fuente: IBGE: Perfil dos Municípios Brasileiros - Meio Ambiente (IBGE, 2005a) y elaboración propia.

Cuadro 10 - Acciones de gestión ambiental (equipos para la gestión)

\begin{tabular}{|c|c|c|c|c|}
\hline $\begin{array}{l}\text { Acciones } \\
\text { de gestión } \\
\text { ambiental: } \\
\text { equipos }\end{array}$ & Equipo & $\begin{array}{c}\text { Porcentaje } \\
\text { de ocurrencia } \\
\text { (ciudades pobres } \\
\text { del } \mathrm{ACP}, \mathrm{N}=20 \text { ) }\end{array}$ & $\begin{array}{c}\text { Porcentaje } \\
\text { de ocurrencia } \\
\text { (ciudades ricas del } \\
\text { ACP, } \mathbf{N}=\mathbf{2 0} \text { ) }\end{array}$ & $\begin{array}{c}\text { Porcentaje de } \\
\text { ocurrencia (universo } \\
\text { de las ciudades } \\
\text { investigadas, } \mathbf{N}=\mathbf{2 8 3} \text { ) }\end{array}$ \\
\hline & $\begin{array}{l}\text { Sitios para recibimiento de } \\
\text { envases de pesticidas }\end{array}$ & Cero & $35 \%$ & $21 \%$ \\
\hline & Vertederos industriales & Cero & $20 \%$ & $12 \%$ \\
\hline & Vertederos sanitarios & $55 \%$ & $65 \%$ & $58 \%$ \\
\hline & $\begin{array}{l}\text { Estaciones de medición de la } \\
\text { calidad del aire }\end{array}$ & Cero & $15 \%$ & $11 \%$ \\
\hline & $\begin{array}{l}\text { Unidades de conservación } \\
\text { municipales }\end{array}$ & $15 \%$ & $85 \%$ & $59 \%$ \\
\hline
\end{tabular}

Fuente: IBGE: Perfil dos Municípios Brasileiros - Meio Ambiente (IBGE, 2005a) y elaboración propia. 
de gestión ambiental deficitario esbozado por los datos del IBGE evidentemente ha traído consecuencias socio-ambientales relevantes. El Cuadro 11 resume algunos de esos impactos.

Gestión ambiental de las ciudades medias: ni siquiera una sostenibilidad blanda

Muchos de los problemas ambientales urbanos resultan de administraciones ineficaces, y de la falta de planificación y políticas urbanas coherentes, más que del proceso de urbanización per se (Hardoy et al., 2001), y en las ciudades brasileñas la gestión y planificación todavía no han sido herramientas ambientalmente efectivas (Leitmann, 1995; Angeoletto, 2008; Angeoletto, 2012).

Algunos economistas y académicos argumentan que el crecimiento económico acaba por generar un considerable volumen de recursos para gastos en políticas de protección ambiental (Leveson-Gower, 1997, critica esta argumentación de modo contundente), pero los resultados de nuestra investigación señalan un cuadro distinto, ya que las acciones de gestión ambiental en las ciudades medias que hemos investigado, de manera general, son débiles y de eficacia discutible. Esto ocurre no solo en las ciudades pobres, con fuertes demandas sociales que disminuyen aún más los pocos recursos disponibles, sino también en las ciudades medias ricas del sur y sureste de Brasil, donde esas acciones son más frecuentes.

Efectivamente, según Dodds (1997), la relación entre el aumento de la renta y una mayor demanda por calidad ambiental ha sido establecida solamente para un conjunto limitado de cuestiones ambientales (por ejemplo bajos niveles de contaminantes en el aire y agua), en ciudades de naciones ricas. Es aquello que se suele denominar sostenibilidad blanda

Todas las ciudades medias están ubicadas en biomas de megabiodiversidad. De estas, destacamos aquellas ubicadas en los biomas Mata Atlântica y Cerrado, que son hotspots de biodiversidad, es decir, biomas de elevada diversidad biológica, que contienen un largo número de especies endémicas y que se encuentran bajo extrema amenaza (por factores como urbanización, agricultura y otros) (Mittermeier et al., 1998).

Son 28 las ciudades apuntadas por el análisis de componentes principales ubicadas en los hotspots de biodiversidad. Además, siete de las ciudades del ACP están ubicadas en zonas de transición entre biomas, los ecotonos, por lo que, desde el punto de vista de la conservación de la diversidad biológica, son especialmente relevantes. Son ellas Arapiraca (en la transición entre los biomas Mata Atlântica y Caatinga); Timon (Cerrado / Caatinga) Americana, Bauru, Botocatu, Rio Claro y São José do Rio Preto (Mata Atlântica / Cerrado); y Santa Cruz do Sul (Mata Atlântica / Pampa).

Smith et al. (1997) estudiaron poblaciones de especies comunes de pájaros de un bosque pluvial y de un ecótono (bosque pluvial / sabana), detectando diferencias morfológicas entre las poblaciones del bosque y del ecotono, a pesar del intenso flujo génico entre ellas. Esos datos evidencian la posibilidad de que los ecotonos sean sitios de especiación, por lo que se recomienda una mayor atención a la conservación de esas zonas de transición (Smith et al., 2001). Así que, para las ciudades medias ubicadas en ecotonos, habría que se destinar recursos para proyectos de investigación aplicables a la planificación y gestión

Cuadro 11 - Ocurrencia de impactos ambientales

\begin{tabular}{lccc}
\hline \multicolumn{1}{c}{$\begin{array}{c}\text { Tipo de impacto } \\
\text { ambiental }\end{array}$} & $\begin{array}{c}\text { Porcentaje de } \\
\text { ocurrencia (ciudades } \\
\text { pobres del ACP, N=20) }\end{array}$ & $\begin{array}{c}\text { Porcentaje de } \\
\text { ocurrencia (ciudades } \\
\text { ricas del ACP, N=20) }\end{array}$ & $\begin{array}{c}\text { Porcentaje de } \\
\text { ocurrencia (universo } \\
\text { de las ciudades } \\
\text { investigadas, N=283) }\end{array}$ \\
\hline Recursos naturales afectados & $85 \%$ & $90 \%$ & $94 \%$ \\
Áreas protegidas afectadas & $65 \%$ & $50 \%$ & $56,2 \%$ \\
Cambios negativos en el paisaje & $80 \%$ & $60 \%$ & $62,2 \%$ \\
\hline
\end{tabular}

Fuente: IBGE: Perfil dos Municípios Brasileiros - Meio Ambiente (IBGE, 2005a) y elaboración propia. 
ambiental urbanas, y que redundasen en menores impactos a la diversidad biológica de esos ecotonos.

Respecto a las acciones de fiscalización, es especialmente preocupante el bajo porcentaje de ciudades que practican la fiscalización sobre la contaminación atmosférica producida por vehículos; sobre las gasolineras y sobre el uso de fertilizantes químicos y pesticidas. Hay en Brasil más de 35.000 gasolineras. De esas, 8.000 están ubicadas en el estado de São Paulo (Lopes et al., 2010), donde se ubican el 29\% de las ciudades medias de Brasil. La gasolina contiene varios contaminantes, entre ellos, benceno, tolueno, etilbenceno y xilenos (conocidos pela sigla BTEX). Los BTEX son extremamente tóxicos. Causan depresión del sistema nervioso central y son carcinogénicos. La gasolina brasileña posee una peculiaridad: se trata efectivamente de una mezcla de $80 \%$ de gasolina y $20 \%$ de etanol (por fuerza de ley federal). Así que, los BTEX son más solubles en etanol y a su vez, el etanol es muy soluble en agua, lo que aumenta la contaminación, en caso de vertidos (Corseuil \& Marins, 1997; Silva et al., 2002).

La presencia de etanol mezclado con la gasolina retrasa la descomposición de los BTEX por acción de microorganismos, y en casos más graves de vertidos, puede que se tarde años hasta que la calidad del agua se normalice para el consumo humano. Por la poca fiscalización municipal de gasolineras, se supone que los casos de contaminación de aguas subterráneas utilizadas para fines humanos no sean hechos inusuales.

Brasil, país que figura entre los más importantes players de la agroindustria global, es uno de los mayores consumidores de pesticidas del mundo (Faria et al., 2007). Entre 1960 y 2000, el área cultivada en Brasil ha aumentado un $78 \%$, mientras que el consumo de pesticidas ha crecido en $700 \%$ (Spadotto, 2006). Las ventas de pesticidas se concentran principalmente en los estados de Rio Grande do Sul, Paraná y São Paulo (Alves, 2002), donde están ubicadas el $44 \%$ de las ciudades medias brasileñas, y 15 de las 20 ciudades ricas señaladas por el ACP.

Los bajos porcenajes de ciudades con sitios apropiados para la recogida de envases usados de pesticidas (ninguna de las ciudades pobres señaladas por el ACP los poseen, aunque el consumo de pesticidas sea significativo en $65 \%$ de ellas), y la poca fiscalización del uso de esos venenos, como también en el caso de las gasolineras, presupone impactos al ambiente y a la salud pública. Por desgracia, los órganos públicos responsables por la fiscalización del uso de pesticidas son débiles, y no presentan condiciones siquiera para reunir y evaluar dados sobre su consumo (Alves, 2002).

Es también digna de mención la poca prioridad a la conservación y recuperación del suelo agrícola en las ciudades medias de Brasil, cuando se observa la dimensión mundial de ese impacto. Globalmente el promedio de erosión está entre 30 a 40 toneladas por hectárea, es decir, la erosión de suelos agrícolas es cerca de 30 a 40 veces más rápida que la tasa natural de formación y reposición del suelo (Pimentel et al., 2010). A pesar de ser un proceso natural, la erosión es notablemente acelerada por actividades humanas, y suelos de regiones tropicales y subtropicales son particularmente propensos a la erosión, porque las lluvias suelen ser más intensas y abundantes (PNUMA, 2011).

Se configura así un cuadro de gestión ambiental de la producción agrícola inconsistente y poco riguroso en las ciudades medias brasileñas. Es poco probable que los productores agrícolas se inhiban en exagerar el uso de pesticidas, si les parece adecuado hacerlo, y la escasa presencia de sitios para la recogida de envases de pesticidas presupone un factor más de contaminación del suelo, agua y aire.

En este contexto, suena irónico que programas de recogida selectiva de basura reciclable o de incentivo a la agricultura orgánica no estén tan presentes en las ciudades medias de Brasil como los programas de educación ambiental. No hay duda que la educación ambiental es una herramienta necesaria a la resolución de nuestros dilemas ambientales. Sin embargo, es frecuente el discurso de la educación ambiental como una panacea para los problemas del medio ambiente. A través de ese discurso, la etiqueta educación ambiental ha sido hábilmente apropiada por los poderes públicos. Problemas como contaminación, gestión de desechos y deforestación son frecuentemente presentados por las autoridades con "acciones de educación ambiental". Es esta una de las vacas sagradas de la preservación ambiental: un argumento políticamente correcto y consensual, pero que genera una visión simplista del medio ambiente, que poco, o nada contribuye a la resolución de nuestros dilemas ecológicos (Angeoletto \& Martins, 2010).

En un momento de descentralización política de ámbito mundial, crecen los servicios y responsabilidades (incluso de orden ambiental) que asumen las ciudades, pero raramente las municipalidades tienen personal 
preparado, y capacidad técnica y administrativa para ellos. Por ello, hay que echar mano de ideas innovadoras y poco costosas, e implantarlas, a través del capital humano disponible. No nos referimos solamente al personal técnico de los ayuntamientos de las ciudades medias, sino también a la participación popular en la planificación y desarrollo de los proyectos. Además, la escasez de funcionarios en las secretarias municipales de medio ambiente de los ayuntamientos puede ser resuelta, por lo menos en parte, con la inclusión de técnicos de otras instituciones, como las universidades.

La planificación y gestión adecuadas de las ciudades son tan importantes para la conservación de la biodiversidad comoo es el establecimiento de áreas naturales legalmente protegidas (Botkin \& Beveridge, 1997; PNUMA, 2011). De hecho, aunque el porcenaje de áreas protegidas esté aumentando mundialmente desde 1990, el número de especies amenazadas sigue creciendo (PNUMA, 2011), hechos que ponen de relieve la importancia de la planificación y gestión urbanas orientadas a la disminución de impactos ambientales causados por las ciudades.

Como cantó el bardo Bob Dylan, beauty walks a razor's edge. Queda claro que, si bien las cuestiones ambientales han alcanzado importancia en la sociedad civil, poca prioridad es dada a los problemas ambientales en el ámbito municipal en Brasil. Ni siquiera hemos alcanzado, en las ciudades medias brasileñas, los estándares de sostenibilidad blanda de ciudades de países desarrollados (es decir, sostenibilidad para unos pocos parámetros, como los relativos a la calidad del agua y aire en ambientes urbanos). ¿Cuándo habrá de hecho una agenda para las cuestiones medioambientales en el ámbito municipal, en Brasil? Hay mucho que hacer, para que no repitamos en las ciudades medias las innumerables tragedias ambientales omnipresentes en las metrópolis brasileñas.

\section{Agradecimientos}

A CAPES, y al Ministerio de Agricultura, Alimentación y Medio Ambiente del gobierno español, por la financiación al Proyecto Ecología Urbana en Regiones Metropolitanas de Brasil: Paisaje, Calidad de Vida y Desarrollo Humano [Plan Nacional de I+D+i (CSO2009-12689)]. A Camila Essy, por la revisión del texto, y al Doctor Marciano Lopes e Silva (in memoriam).

\section{Referencias}

Alves, J. P., Fo. (2002). Uso de agrotóxicos no Brasil: controle social e interesses corporativos. São Paulo: Annablume.

Angeoletto, F. (2008). Pelos quintais de Sarandi: ecologia urbana e planejamento ambiental. Maringá: EDUEM.

Angeoletto, F. (2012). Planeta ciudad: ecología urbana y planificación de ciudades medias de Brasil (Tesis doctoral). Departamento de Ecología, Universidad Autónoma de Madrid, Madrid.

Angeoletto, F., \& Martins, N. (2010). Vacas Sagradas: os mitos nos discursos dos militantes ambientalistas. Revista Travessias, 8, 357-370.

Barrasa, S. (2007). El paisaje en América Latina: experiencia de valoración participada de paisajes visuales para la planificación ambiental de La Habana - Cuba (Tesis doctoral). Departamento de Ecología, Universidad Autónoma de Madrid, Madrid.

Bettini, V. (1998). Elementos de ecología urbana. Madrid: Editorial Trotta.

Botkin, D. B., \& Beveridge, C. E. (1997). Cities as environments. Urban Ecosystems, 1(1), 3-19. http://dx.doi. org/10.1023/A:1014354923367.

Brasil. Ministério das Cidades. (2004). Perfil da mobilidade, do transporte e do trânsito nos municípios brasileiros. Brasília: Ministério das Cidades.

Carvalho, E. (2003). Exclusão social e crescimento das cidades médias brasileiras. Scripta Nova - Revista Electrônica de Geografía y Ciencias Sociales, 6(146), 70-83.

Chapin, F. III., Carpenter, S., Kofinas, G. P., Folke, C., Abel, N., Clark, W. C., Olsson, P., Smith, D. M., Walker, B., Young, O. R., Berkes, F., Biggs, R., Grove, J. M., Naylor, R. L., Pinkerton, E., Steffen, W., \& Swanson, F. J. (2010). Ecosystem stewardship: sustainability strategies for a rapidly changing planet. Trends in Ecology \& Evolution, 25(4), 241-249. http:// dx.doi.org/10.1016/j.tree.2009.10.008. PMid:19923035.

Cohen, J. E. (2003). Human population: the next half century. Science, 302(5648), 1172-1175. http://dx.doi. org/10.1126/science.1088665. PMid:14615528.

Cohen, J. E. (2005). A maturidade da população. Scientific American Brasil, 41, 40-47.

Comisión Econômica para América Latina y el Caribe de las Naciones Unidas - CEPAL. (2000). De la urbanización 
acelerada a la consolidación de los asentamientos humanos en América Latina y el Caribe: el espacio regional. Santiago: CEPAL.

Corseuil, H. X., \& Marins, M. D. M. (1997). Contaminação de águas subterrâneas por derramamento de gasolina: o problema é grave? Revista Engenharia Sanitária e Ambiental, $2(2), 50-54$.

Dodds, S. (1997). Economic growth and human well-being. In M. Diesendorf, \& C. Hamilton (Eds.). Human ecology, human economy (p. 48-55). Sidney: Allen \& Unwin.

Faria, N. M., Fassa, A. C., \& Facchini, L. (2007). Intoxicação por agrotóxicos no Brasil: os sistemas oficiais de informação e desafios para realização de estudos epidemiológicos. Ciencia \& Saude Coletiva, 12(1), 25-38. http://dx.doi.org/10.1590/ S1413-81232007000100008. PMid:17680056.

Garza, G. (1996). Uncontrolled air pollution in Mexico City. Cities (London, England), 13(5), 315-328. http://dx.doi. org/10.1016/0264-2751(96)00019-4.

Grimm, N. B., Faeth, S. H., Golubiewski, N. E., Redman, C. L., Wu, J., Bai, X., \& Briggs, J. M. (2008). Global change and the ecology of cities. Science, 319(5864), 756-760. http:// dx.doi.org/10.1126/science.1150195. PMid:18258902.

Hardoy, J. E., Mitlin, D., \& Satterthwaite, D. (2001). Environmental problems in a urbanizing world: finding solutions in Africa, Asia and Latin America. London: Earthscan.

Instituto Brasileiro de Geografia e Estatística - IBGE. (2005a). Perfil dos municípios brasileiros: meio ambiente, 2002. Rio de Janeiro, Brasil.

Instituto o Brasileiro de Geografia e Estatística - IBGE. (2005b). Pesquisa de Informações Básicas Municipais. Perfil dos Municípios Brasileiros. Rio de Janeiro: IBGE. Recuperado el 11 de abril de 2014, de http://www.ibge.gov.br/home/ estatistica/economia/perfilmunic/meio_ambiente_2002/ meio_ambiente2002.pdf

Instituto Brasileiro de Geografia e Estatística - IBGE. (2015). Mapas, bases e referenciais, bases cartográficas e cartas.Rio de Janeiro: IBGE. Recuperado el 07 de febrero de 2015, de http://mapas.ibge.gov.br/bases-e-referenciais/ bases-cartograficas/cartas.html

Instituto o Brasileiro de Geografia e Estatística - IBGE. (2014).Cidades@. Rio de Janeiro: IBGE. Recuperado el 09 de septiembre de 2014, de http://www.cidades.ibge. gov.br/xtras/home.php
Instituto de Pesquisa Econômica Aplicada - IPEA. (2011). Comunicados do IPEA, $n$ - 68: análise preliminar dos dados do Censo 2010. Brasília, IBGE.

Instituto de Terras, Cartografia e Geociências - ITCG. (2015). Plano cartográfico estadual. Curitiba: ITCG. Recuperado el 17 de febrero de 2015, de http://www.itcg.pr.gov.br/ modules/conteudo/conteudo.php?conteudo $=9$

Kasanko, M., Barredo, J., Lavalle, C., McCormick, N., Demicheli, L., Sagris, V., \& Brezger, A. (2006). Are European cities becoming dispersed? A comparative analysis of 15 European urban areas. Landscape and Urban Planning, 77(1), 111130. http://dx.doi.org/10.1016/j.landurbplan.2005.02.003.

Keilman, N. (2003). Biodiversity: the threat of small households. Nature, 421(6922), 489-490. http://dx.doi. org/10.1038/421489a. PMid:12556874.

Leitmann, J. (1995). A global synthesis of seven urban environmental profiles. Cities (London, England), 12(1), 23-39. http://dx.doi.org/10.1016/0264-2751(95)91863-B.

Leveson-Gower, H. (1997). Trade and the environment. In M. Diesendorf, C. Hamilton (Eds.). Human ecology, human economy. Sydney: Allen \& Unwin Publishers.

Liu, J., Daily, G. C., Ehrlich, P. R., \& Luck, G. W. (2003). Effects of household dynamics on resource comsuption and biodiversity. Nature, 421(6922), 530-533. http://dx.doi. org/10.1038/nature01359. PMid:12540852.

Lopes, M. B., Silva, A. L., \& Conejero, M. A. (2010). Fluxos e poder nos canais de distribuição de etanol carburante: um estudo qualitativo no estado de São Paulo. Revista de Administração, 45(4), 356-372.

Martins, L. C., Latorre, M. R. D. O., Cardoso, M. R. A., Goncalves, F. L., Saldiva, P. H., \& Braga, A. L. (2002). Poluição atmosférica e atendimentos por pneumonia e gripe em São Paulo, Brasil. Revista de Saude Publica, 36(1), 88-94. http://dx.doi.org/10.1590/S0034-89102002000100014. PMid:11887235.

Martins, L. C., Latorre, M. R. D. O., Saldiva, P. H. N., \& Braga, A. L. F. (2001). Relação entre poluição atmosférica e atendimentos por infecção de vias aéreas superiores no município de São Paulo: avaliação do rodízio de veículos. Revista Brasileira de Epidemiologia, 4(3), 220-229. http:// dx.doi.org/10.1590/S1415-790X2001000300008.

Mega, V. (2000). Cities inventing the civilisation of sustainability: an odyssey in the urban archipelago of the European Union. Cities, 17(3), 227-236. 
Mittermeier, R. A., Myers, N., Thomsen, J. B., Da Fonseca, G. A., \& Olivieri, S. (1998). Biodiversity hotspots and major tropical wilderness areas: approaches to setting conservation priorities. Conservation Biology, 12(3), 516-520. http:// dx.doi.org/10.1046/j.1523-1739.1998.012003516.x.

Oliveira, C., \& Leitmann, J. (1994). São Paulo: environmental profile. Cities (London, England), 11(1), 10-14. http:// dx.doi.org/10.1016/0264-2751(94)90044-2.

Pickett, S. T. A., Burch, W. R. Jr., Dalton, S. E., \& Foresman, T. W. (1997). Integrated urban ecosystem research. Urban Ecosystems, 1(4), 183-184. http://dx.doi. org/10.1023/A:1018579628818.

Pimentel, D., Whitecraft, M., Scott, Z. R., Zhao, L., Satkiewicz, P., Scott, T. J., Phillips, J., Szimak, D., Singh, G., Gonzalez, D. O., \& Moe, T. L. (2010). Will Limited Land, Water, and Energy Control Human Population Numbers in the Future? Human Ecology, 38(5), 599-611. http://dx.doi.org/10.1007/ s10745-010-9346-y.

Prado, P. I., Lewinsohn, T. M., Carmo, R. L., \& Hogan, D. J. (2001). Ordenação Multivariada na Ecologia e seu Uso em Ciências Ambientais. Ambiente e Sociedade, 10, 69-83.

Programa de las Naciones Unidas para el Medio Ambiente - PNUMA.(2011). Anuario PNUMA: temas emergentes en nuestro medio ambiente global. Nairobi: PNUMA.

Rees, W. E. (1997). Urban ecosystems: the human dimension. Urban Ecosystems, 1(1), 63-75. http://dx.doi. org/10.1023/A:1014380105620.

Saldiva, P. H. N., Lichtenfels, A. J. F. C., Paiva, P. S. O., Barone, I. A., Martins, M. A., Massad, E., Pereira, J. C. R., Xavier, V. P., Singer, J. M., \& Bohm, G. M. (1994). Association between air pollution and mortality due to respiratory diseases in children in São Paulo, Brazil: a preliminary report. Environmental Research, 65(2), 218-225. http://dx.doi. org/10.1006/enrs.1994.1033. PMid:8187738.

Silva, J. M. C., Tabarelli, M., Fonseca, M. T., \& Lins, L. V. (2003). Biodiversidade da caatinga: áreas e ações prioritárias para a conservação. Brasília: Ministério do Meio Ambiente.

Silva, R. L. B., Barra, C. M., Monteiro, T. C. N., \& Brilhante, O. M. (2002). Estudo da contaminação de poços rasos por combustíveis orgânicos, e possíveis conseqüências para a saúde pública ao município de Itaguaí, Rio de Janeiro, Brasil. Cadernos de Saúde Pública, 18(6), 1599-1607. http://dx.doi.org/10.1590/S0102-311X2002000600014. PMid:12488887.
Smith, T. B., Kark, S., Schneider, C. J., Wayne, R. K., \& Moritz, C. (2001). Biodiversity hotspots and beyond: the need for preserving environmental transitions. Trends in Ecology \& Evolution, 16(8), 431. http://dx.doi.org/10.1016/ S0169-5347(01)02201-7.

Smith, T. B., Wayne, R. K., Girman, D. J., \& Bruford, M. W. (1997). A role for ecotones in generating rainforest biodiversity. Science, 276(5320), 1855-1857

Spadotto, C. A. (2006). Avaliação de riscos ambientais de agrotóxicos em condições brasileiras. Embrapa Meio Ambiente: Documentos, 58, 2-22.

Terradas, J.(2001). Ecología urbana. Barcelona: Editorial Rubes.

United Nations Economic Commission for Latin America and the Caribbean - ECLAC. United Nations Environment Programme - UNEP. (2001). The sustainbility of developtment in Latin America and the Caribbean: challenges and opportunities. Santiago: ECLAC/ UNEP.

United Nations Human Settlements Programme - UNHABITAT. (2008). State of the world's cities 2008/2009. London: Earthscan.

United Nations Population Fund - UNFPA. (2007). Estado de la población mundial 2007. New York: United Nations Population Fund.

United Nations. Department of Economic and Social Affairs/Population Division. (2008). World urbanization prospects: the 2007 revision. New York: Department of Economic and Social Affairs.

Vianna, M. M. B., Portugal, L. S., \& Balassiano, R. (2004). Intelligent transportation systems and parking management: implementation potential in a Brazilian city. Cities (London, England), 21(2), 137-148. http://dx.doi.org/10.1016/j. cities.2004.01.001.

Vitousek, P. M. (1994). Beyond global warming: ecology and global change. Ecology, 75(7), 1861-1876. http:// dx.doi.org/10.2307/1941591.

WorldWatch Institute. (2006). Estado do Mundo 2005: estado do consumo e consumo sustentável. Salvador: UMA.

Recibido: Abr. 12, 2015

Aprobado: Jul. 23, 2015 